\title{
Hyperoside suppresses BMP-7-dependent PI3K/AKT pathway in human hepatocellular carcinoma cells
}

\author{
Shuang Wei ${ }^{1 \#}$, Yun Sun ${ }^{1 \#, ~ L i ~ W a n g ~}{ }^{1 \#}$, Tianfang Zhang ${ }^{1}$, Wendi Hu ${ }^{2}$, Wangxiao Bao ${ }^{1}$, Lin Mao ${ }^{1}$, \\ Jinxiu Chen ${ }^{1}$, Haijun $\mathrm{Li}^{1}$, Yankai Wen ${ }^{3}$, Zuobing Chen ${ }^{1}$ \\ ${ }^{1}$ Department of Rehabilitation Medicine, The First Affiliated Hospital, College of Medicine, Zhejiang University, Hangzhou, China; ${ }^{2}$ Division of \\ Hepatobiliary and Pancreatic Surgery, Department of Surgery, The First Affiliated Hospital, College of Medicine, Zhejiang University, Hangzhou, \\ China; ${ }^{3}$ Department of Anesthesiology, McGovern Medical School, University of Texas Health Science Center at Houston, Houston, TX, USA \\ Contributions: (I) Conception and design: Z Chen, S Wei, Y Wen, L Wang; (II) Administrative support: Z Chen; (III) Provision of study materials or \\ patients: T Zhang, W Hu; (IV) Collection and assembly of data: L Mao, H Li, J Chen; (V) Data analysis and interpretation: S Wei, Y Sun, W Bao; (VI) \\ Manuscript writing: All authors; (VII) Final approval of manuscript: All authors. \\ \#These authors contributed equally to this work. \\ Correspondence to: Zuobing Chen. Department of Rehabilitation Medicine, The First Affiliated Hospital, College of Medicine, Zhejiang University, \\ Qingchun Road No. 79, Hangzhou, China. Email: czb1971@zju.edu.cn.
}

\begin{abstract}
Background: New therapeutics for hepatocellular carcinoma (HCC) are urgently needed and searching for new anti-cancer compounds in plant medicines may represent a promising approach. The present study was conducted to clarify the role of hyperoside (HP) and its underlying molecular mechanism in a cancer cell.

Methods: Bone morphogenetic protein 7 (BMP-7) protein expression was measure in Human HCC tissue. In in vitro experiments, HP effects on cell proliferation and the mechanism were investigated deeply.

Results: The result showed a higher expression of BMP-7 in human HCC compared to adjacent noncancerous counterparts, and that silencing of BMP-7 suppressed HepG2 cell proliferation, suggesting BMP-7 plays an anti-cancer role in HCC. Furthermore, we found that HP could induce cell cycle arrest in proliferating HepG2 cells at the G1 phase by decreasing BMP-7 expression and that the phosphorylation of AKT and expression of PI3K were significantly down-regulated upon treatment of HP or BMP-7 knockdown. In addition, silencing of BMP-7 abrogated the difference of AKT phosphorylation between cells with and without HP treatment.

Conclusions: Our results indicated that HP suppressed cell proliferation by inhibiting the BMP-7dependent PI3K/AKT signaling pathway in HepG2 HCC cells, and either HP supplement or targeting BMP-7 might be a promising treatment against HCC.
\end{abstract}

Keywords: Hepatocellular carcinoma (HCC); hyperoside (HP); bone morphogenetic protein 7 (BMP-7); cell proliferation; AKT

Submitted Apr 02, 2021. Accepted for publication Jul 29, 2021.

doi: 10.21037/atm-21-2980

View this article at: https://dx.doi.org/10.21037/atm-21-2980

\section{Introduction}

Hepatocellular carcinoma (HCC) is the fourth leading cause of cancer-related death worldwide, accounting for approximately $8.3 \%$ of all cancers (1). Despite therapeutic approaches for patients with HCC having progressed rapidly over the past decades, the mortality rate remains high (2). One of the main reasons for the high mortality among
HCC patients is the lack of effective treatments to deal with the complicated molecular pathogenesis of HCC (3). Thus, it is urgent to discover new molecular targets and develop new therapeutics for the disease.

Bone morphogenetic proteins (BMPs) are multifunctional growth factors that belong to the transforming growth factor- $\beta$ (TGF- $\beta$ ) superfamily which regulate cell 
proliferation, apoptosis, and differentiation $(4,5)$. In recent years, increasing studies have suggested that the aberrant expression of BMPs is positively correlated with disease progression and survival in several cancers (6-12). However, the role of BMP-7 in cancers is not well characterized and varies when the environment changes $(13,14)$. For example, while BMP-7 can promote and also inhibit cell growth in breast cancer cells in suitable environments (15) and it has been reported as a prognostic biomarker for HCC (16), its role in HCC development remains elusive.

Plant species of the genus Hypericum are famous for their use in traditional medicine because of their therapeutic efficacy (17). Hyperoside (HP) is one of the flavonoid components of Celastraceae, Ericaceae and Guttifera, and has been reported to exert anti-cancer effects $(18,19)$. Nevertheless, studies about their effect on HCC and its underlying mechanisms remain elusive. In the current study, we hypothesized that HP inhibits HCC cell proliferation, which may be associated with the down-regulation of BMP-7 expression and blockage of the PI3K/AKT signaling pathway. Our findings support a novel role of HP, as an effective modulator of BMP7 expression and finally a potential therapeutic choice in anti-cancer activity. We present the following article in accordance with the MDAR reporting checklist (available at https://dx.doi.org/10.21037/ atm-21-2980).

\section{Methods}

\section{Reagents and antibodies}

HP was purchased from Selleck (S5453, Houston, TX, USA) and dimethyl sulfoxide (DMSO, D4540) and thiazolyl blue tetrazolium bromide (MTT, M5655) were purchased from Sigma (St. Louis, MO, USA). Mouse monoclonal antibodies $\beta$-actin (BS6007M) was purchased from Bioworld Technology (Bloomington, MN, USA), and rabbit anti-AKT (9272), rabbit anti-PI3K (4249), and rabbit anti-phosphorylated AKT (P-AKT, 4060) polyclonal antibody from Cell Signaling Technology (Danvers, MA, USA). Mouse anti-BMP-7 (sc53917) was purchased from Santa Cruz (Dallas, TX, USA) and rabbit anti-Cyclin D1 (ab226977) polyclonal antibody and rabbit anti-c-Myc (ab9106) polyclonal antibody from Abcam (Cambridge, UK).

\section{Tissue samples}

Human HCC tissue samples were acquired from three
HCC patients who underwent liver resection surgery at the Department of Surgery, The First Affiliated Hospital, College of Medicine, Zhejiang University (Hangzhou, China). The patient's adjacent noncancerous tissues $(>2 \mathrm{~cm}$ away from the tumor) served as the control group in this study. All procedures performed in this study were in accordance with the Declaration of Helsinki (as revised in 2013). Clinical Research Ethics Committee of the First Affiliated Hospital, College of Medicine, Zhejiang University approved the design of the study (IIT20210), and the patient sample study protocol. All patients provided signed informed consent.

\section{Histopathology}

The liver tissues were fixed in 4\% paraformaldehyde for at least 24 hours then processed and embedded in paraffin. Five $\mu \mathrm{m}$ thick sections were then stained with hematoxylin and eosin (H\&E).

\section{Cell culture}

Human HepG2 cells were obtained from the Cell Bank of Academy of Sciences (Shanghai, China) and cultured in Dulbecco's modified Eagle's medium (DMEM, Gibco) supplemented with $10 \%$ (vol/vol) heat-inactivated fetal bovine serum (FBS, Gibco) at $37^{\circ} \mathrm{C}$ humidified incubator under $5 \% \mathrm{CO}_{2}$. Cells were cultured by adding different concentrations of HP for 24 hours.

\section{RNA extraction and quantitative real-time polymerase chain reaction}

Total RNA was extracted from cells using TRIzol (Invitrogen) according to the manufacture's instruction, and reverse transcribed to cDNA using a cDNA synthesis kit (TaKaRa). The cDNA was subjected for the quantification of gene expression according to the manufacture's instruction using a SYBR Green PCR Master kit (QIAGEN) and the primers used were listed as follows: BMP-7 (forward: 5 '-TCAACCTCGTGGAACATGACA-3'; reverse: 5 '-CTTGGAAAGATCAAACCGGAACT-3'), $\beta$-actin (forward: 5'-CCCACACTGTGCCCATCTACG-3'; reverse: 5 '-GCCATCTCTTGCTCGAAGTCC-3'). $\beta$-actin served as the internal control.

\section{Western blot}

Cells were lysed in protein extraction solution (Beyotime, 
China) and the protein concentration of each sample was determined by a NanoDrop 2000 Spectrophotometer (Thermo Scientific). Equal amounts of protein were then electrophoresed using SDS-PAGE and blotted onto PVDF membranes (Millipore). After blockade of nonspecific protein binding, membranes were incubated with primary antibodies at $4{ }^{\circ} \mathrm{C}$ overnight, and the primary antibodies recognizing BMP-7, AKT, P-AKT, PI3K, Cyclin D1, c-Myc, and $\beta$-actin were used by a dilution 1:200, 1:500, 1:500, 1:500, 1:500, 1:500, and 1:3,000, respectively. The membranes were then washed and incubated with HRP-conjugated secondary antibodies. Finally, proteins were visualized with an ECLchemiluminescent kit (Thermo Scientific).

\section{Cell cycle analysis}

A Cell Cycle Analysis Kit (Beyotime, China) was used according to the manufacturer's instructions. Analysis was performed on Cytomics FC 500 MCL (Beckman Coulter) and cell populations in the $G_{0} / G_{1}, S$, and $G_{2} / M$ phases were quantified by the Multicycle data analysis software package (Verity Software House).

\section{Immunobistochemistry}

Tissue sections were dehydrated, and antigen retrieval was performed in a microwave oven with inculcation in citrate buffer $(\mathrm{pH}=6.0)$ for $25 \mathrm{~min}$. Sections were then blocked and incubated with primary antibody against BMP-7 (1:200) at $4{ }^{\circ} \mathrm{C}$ overnight. The sections were then washed and incubated with HRP-conjugated secondary antibody for 1 hour at room temperature and the expression of BMP-7 was visualized by 3,3'-diaminobenzidine tetrahydrochloride (DAB) staining.

\section{Cell immunofluorescent staining}

Cells in eight-well culture slides were fixed with acetone for 10 min then blocked and incubated with primary antibody against BMP-7 (1:500) at $4{ }^{\circ} \mathrm{C}$ overnight. Following incubation with FITC-conjugated secondary antibody, the culture slides were counterstained with DAPI and observed using a Leica confocal microscope (Germany).

\section{Statistical analysis}

Results are presented as mean \pm SEM. The result analysis was performed using one-way analysis of variance (ANOVA) or two-tailed unpaired Student's $t$-tests, and $\mathrm{P}<0.05$ was considered statistically significant. At least three independent experiments are performed in every figure.

\section{Results}

\section{Effect of BMP-7 on cell proliferation in human HCC}

BMP-7 has been implicated to be involved in regulating cancer cell proliferation, and to examine whether BMP-7 is involved in HCC, we collected three HCC tissues and their adjacent noncancerous counterparts (referred to as "controls"). H\&E staining showed that human HCC tissues exhibited severe cellular edema and inflammatory infiltration compared to the controls (Figure 1A), and western blot analysis showed that the expression of BMP-7 was higher in HCC tissues compared to the control tissues (Figure $1 B$ ). In addition, immunohistochemistry results showed HCC tissues had more BMP-7 positive staining than their controls (Figure 1C). These data suggest that upregulation of BMP-7 may be involved in the development and progression of HCC.

To investigate the role of BMP-7 in cancer cells, we generated BMP-7 knockdown HepG2 cells using siRNA and found that at 24 hours after transfection, BMP-7 was efficiently silenced in BMP-7-siRNA HepG2 cells (Figure 1D). Notably, BMP-7-siRNA HepG2 cells showed suppressed proliferation in MTT assay (Figure 1E). In summary, our results indicated that BMP-7 played a significant role in the proliferation of HepG2 cells, and that it may be a potential target for human HCC.

\section{$H P$ reduces BMP-7 expression in HepG2 cells}

To investigate the effect of HP on the expression of BMP7 in HepG2 cells, cells were treated with HP (5, 10, 20, 40, and $80 \mu \mathrm{M})$ for 24 hours. The protein and mRNA levels of BMP-7 in the treatment group were significantly decreased compared with the control group in a dose-dependent manner (Figure 2A). Immunofluorescent staining of BMP7 also indicated that BMP-7 was down-regulated in HPtreated HepG2 cells (Figure 2B).

\section{HP suppresses the proliferation and induces cell cycle arrest in HepG2 cells}

To determine the effect of HP on the proliferation of HepG2 cells, cells were treated with $\operatorname{HP}(5,10,20,40$, and 
A
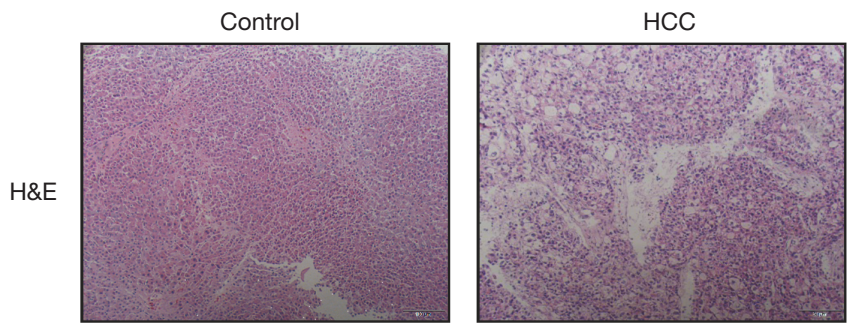

B
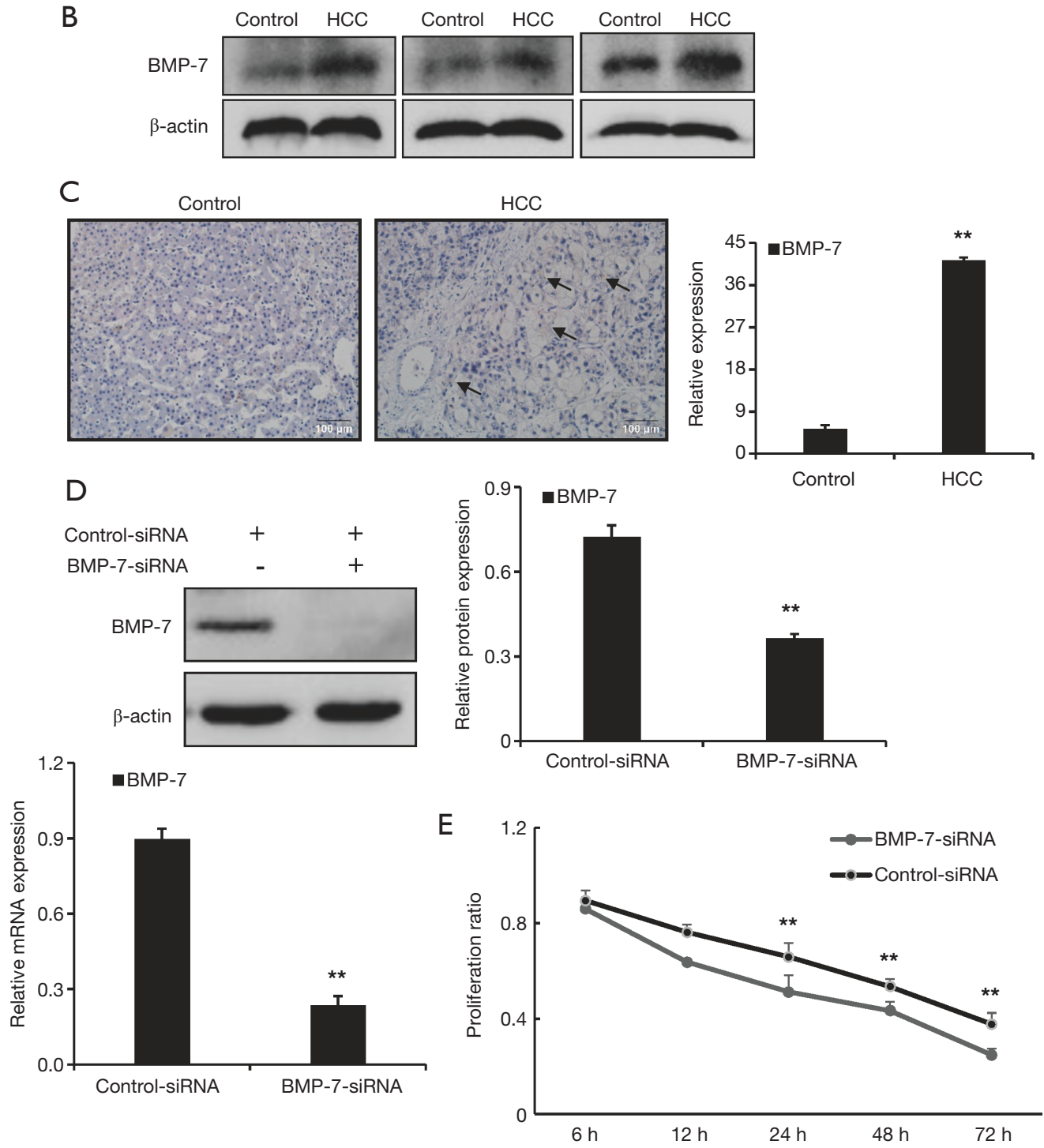

Figure 1 BMP-7 expression and the effect of BMP-7 on cell proliferation in human HCC. (A) H\&E staining was performed in HCC samples and their adjacent noncancerous counterparts (control) (100x magnification). (B) Western bloting result shows the protein expression of BMP-7 in human HCC tissues and controls. (C) Immunohistochemistry of BMP-7 in HCC tissues and controls (200x magnification). (D) BMP-7 knockdown was performed in HepG2 cells, and the mRNA and protein expression of BMP-7 was detected. (E) Effect of BMP-7 on cell proliferation in HepG2 cells. Cell viability was assessed by MTT assay. Data are expressed as mean \pm SEM. **, $\mathrm{P}<0.01$; and at least three independent experiments were performed in each panel. BMP-7, bone morphogenetic protein 7; HCC, hepatocellular carcinoma; H\&E, hematoxylin and eosin; MTT, thiazolyl blue tetrazolium bromide. 

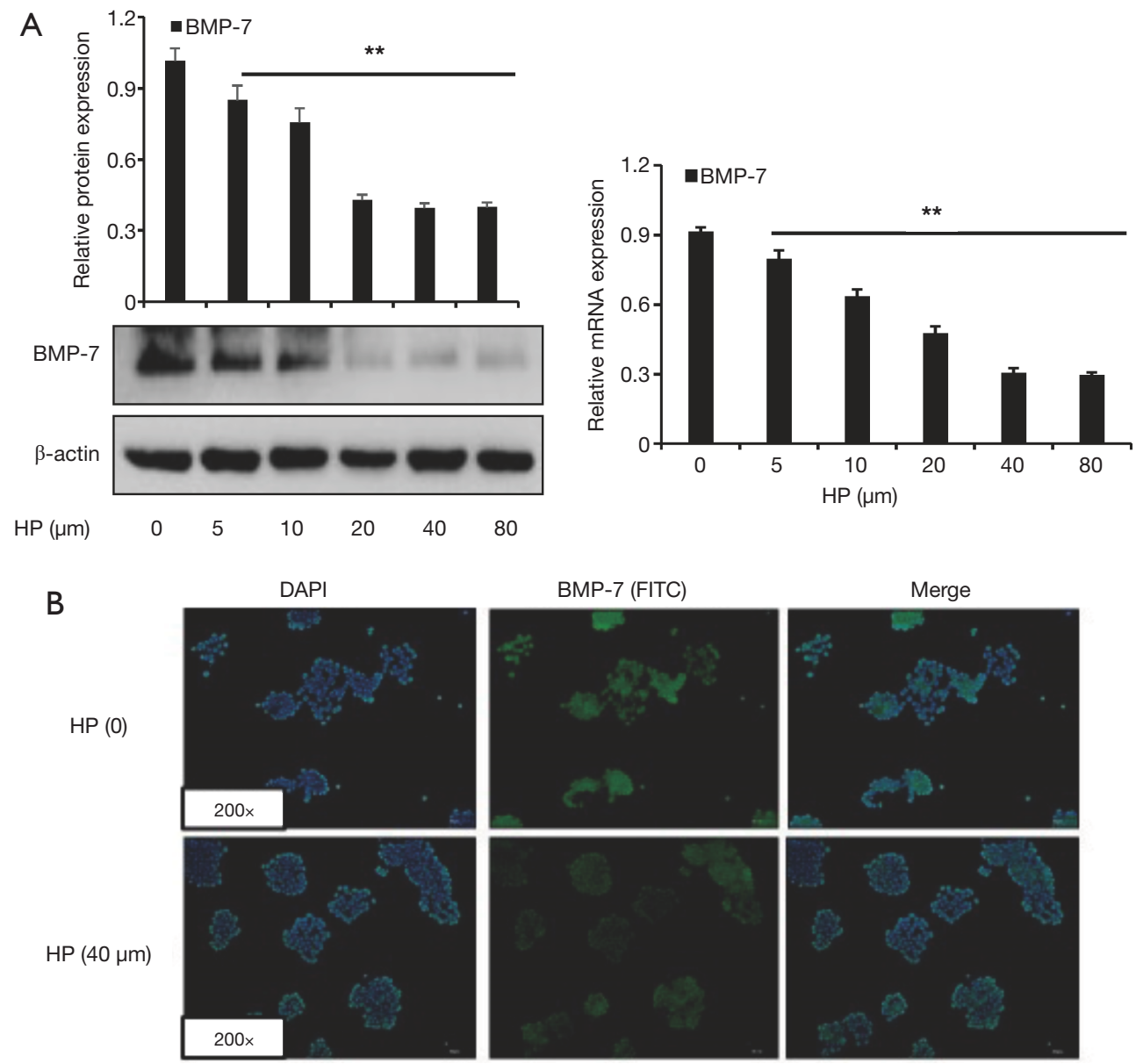

Figure $2 \mathrm{HP}$ reduces BMP-7 expression in HepG2 cells. (A) Cells were treated with HP (5, 10, 20, 40, and 80 $\mu$ M) for 24 hours, the protein and mRNA levels of BMP-7 in the cells were measured by western blot and qPCR, and $\beta$-actin was served as an internal control. (B) Immunofluorescent staining of BMP-7 was performed in HP-treated HepG2 cells and their controls (200× magnification). Data are expressed as mean \pm SEM. ${ }^{* *}, \mathrm{P}<0.01$; and at least three independent experiments were performed in each panel. HP, hyperoside; BMP-7, bone morphogenetic protein 7 .

$80 \mu \mathrm{M})$ for 24 hours, and cell viability was measured by MTT assay. The MTT result showed that HP inhibited cell proliferation by $6.87 \% \pm 0.032 \%, 23.03 \% \pm 0.043 \%$, $47.27 \% \pm 0.035 \%, 65.36 \% \pm 0.020 \%$, and $65.5 \% \pm 0.011 \%$, respectively (Figure 3A), suggesting that HP suppresses HepG2 cell proliferation in a dose-dependent manner.

To investigate the potential mechanism by which HP suppresses the proliferation of HepG2 cells, the cell cycle was analyzed by flow cytometry after HP treatment. The results showed that HP remarkably enhanced the percentage of HepG2 cells in the $G_{0} / G_{1}$ phase compared with that in the control group, and concurrently, cell numbers in the $G_{2} / M$ phase decreased in HP-treated cells (Figure 3B).

Cyclin D1 and c-Myc are involved in the progression of the $G_{1} / S$ phase and were obviously enhanced in human HCC tissues (Figure 3C). However, the expression of Cyclin D1 and c-Myc were significantly reduced in cells under administration of HP (Figure 3D). Taken together, these results suggests that HP inhibits cell proliferation in HepG2 cells through cell cycle arrest.

\section{BMP-7 regulates the PI3K/AKT signaling patbway in HepG2 cells}

The PI3K/AKT signaling pathway plays a pivotal role in fundamental cellular functions such as cancer cell differentiation, cell proliferation, and tissue polarity (20). To determine the effect of BMP-7 on the PI3K/AKT 

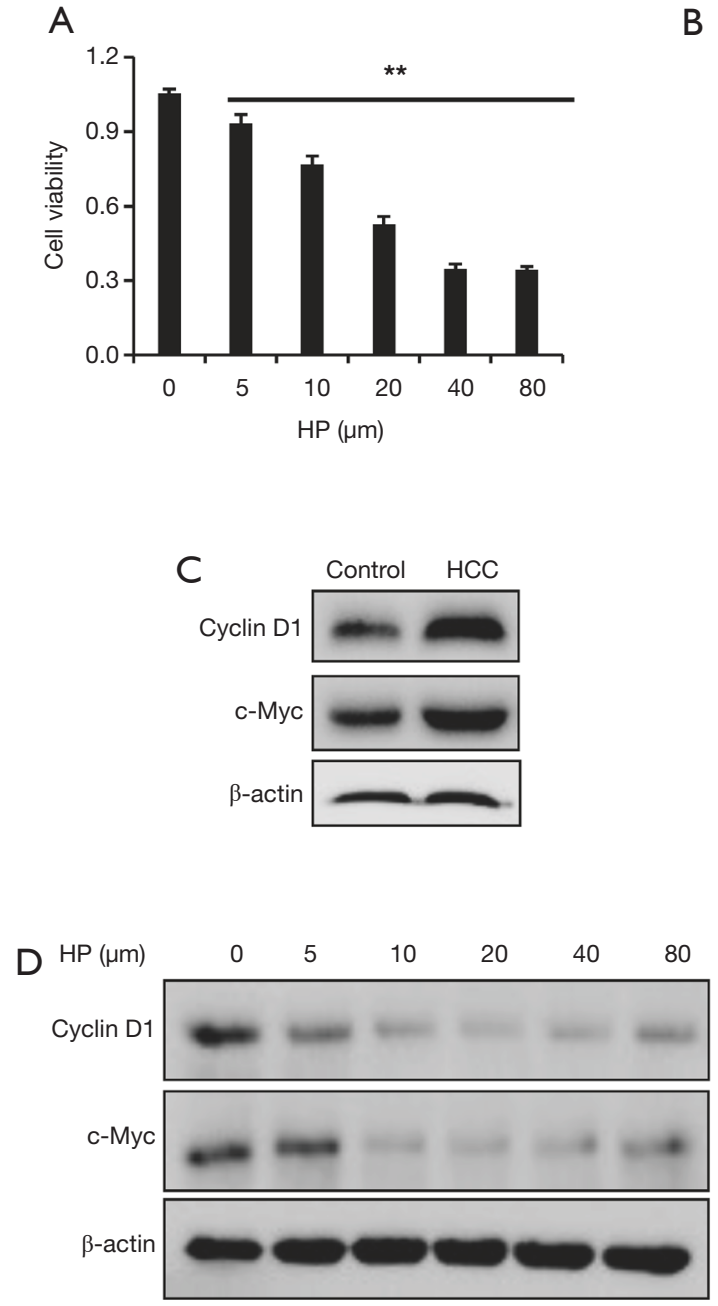
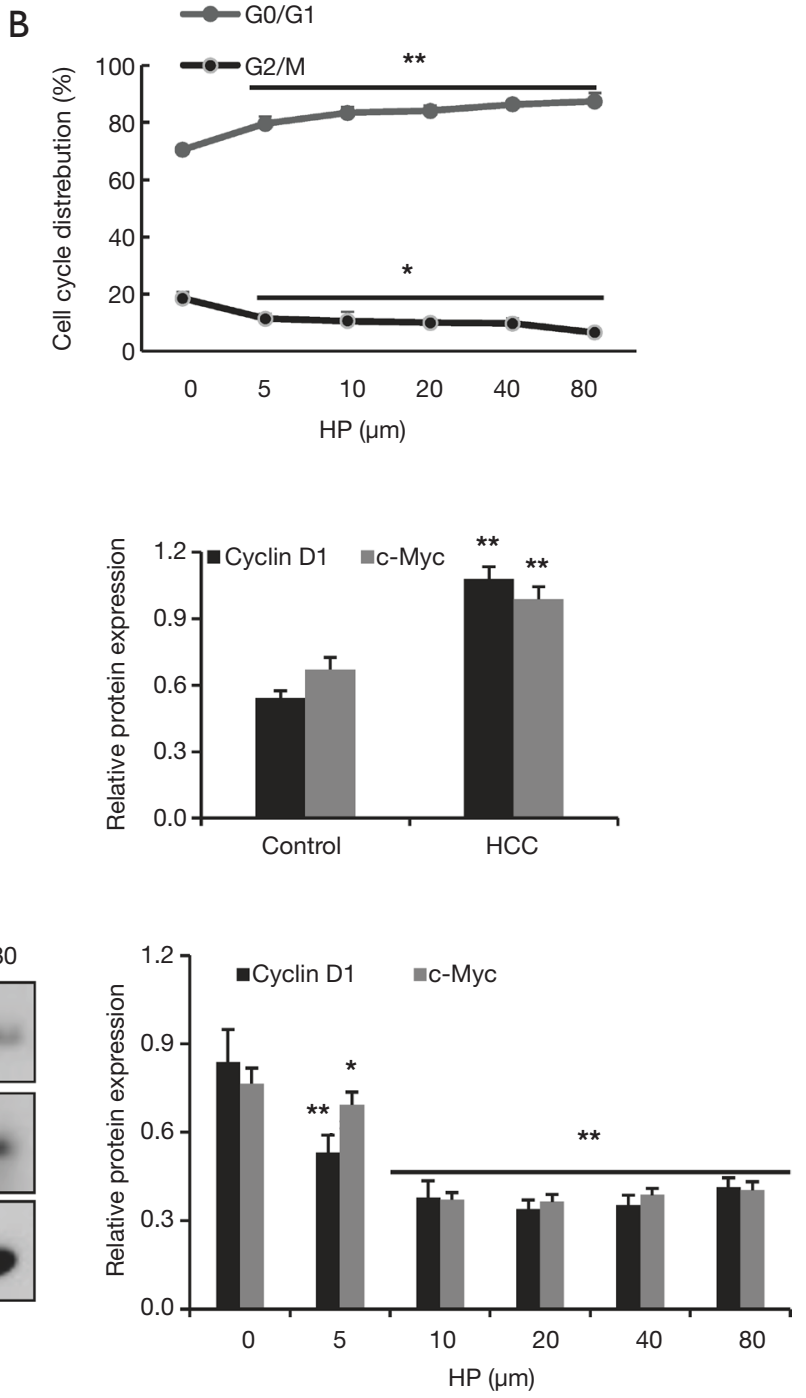

Figure $3 \mathrm{HP}$ suppresses cell proliferation and induces cell cycle arrest. (A) Cell viability was assessed by MTT assay after HP treatment for 24 hours. (B) Flow cytometry for cell cycle analysis was performed in cells treated with HP for 24 hours. (C) The expression of Cyclin D1 and c-Myc were analyzed by western blot in human HCC and controls. (D) The expression of Cyclin D1 and c-Myc were analyzed by western blot in HepG2 cells treated with HP for 24 hours. Data are expressed as mean $\pm \mathrm{SEM} .{ }^{*}, \mathrm{P}<0.05$; ${ }^{* *}, \mathrm{P}<0.01$; and at least three independent experiments were performed in each panel. HP, hyperoside; MTT, thiazolyl blue tetrazolium bromide; HCC, hepatocellular carcinoma.

signaling pathway in HepG2 cells, the levels of P-AKT, AKT, and PI3K were examined, and western blot analysis indicated that PI3K and P-AKT were obviously upregulated in human HCC tissues (Figure $4 A$ ). Furthermore, the protein expression of $\mathrm{P}-\mathrm{AKT}$ and $\mathrm{PI} 3 \mathrm{~K}$ was remarkably down-regulated in cells transfected with BMP-7-siRNA, compared with those in control-siRNA transfected cells (Figure $4 B$ ). These data suggest BMP-7 can regulate the $\mathrm{PI} 3 \mathrm{~K} / \mathrm{AKT}$ signaling pathway in HepG2 cells.

\section{HP inbibits the PI3K/AKT signaling patbway through $B M P 7$ regulation in $\mathrm{HepG} 2$ cells}

Since both HP and BMP-7 knockdown inhibit hepatocellular cancer cell proliferation and given that $\mathrm{HP}$ could attenuate BMP-7 expression in HepG2 cells, we hypothesized that HP inhibited the BMP-7-dependent PI3K/AKT pathway. HepG2 cells were treated with HP for 24 hours, following which the expression of P-AKT and 
A

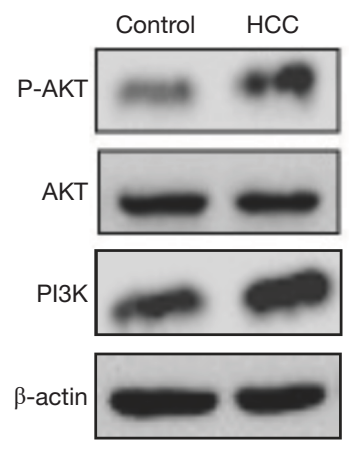

B

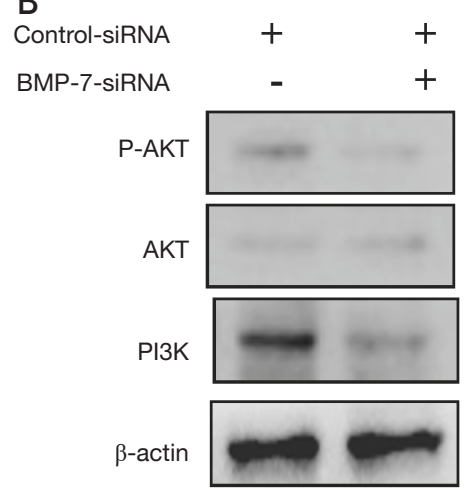

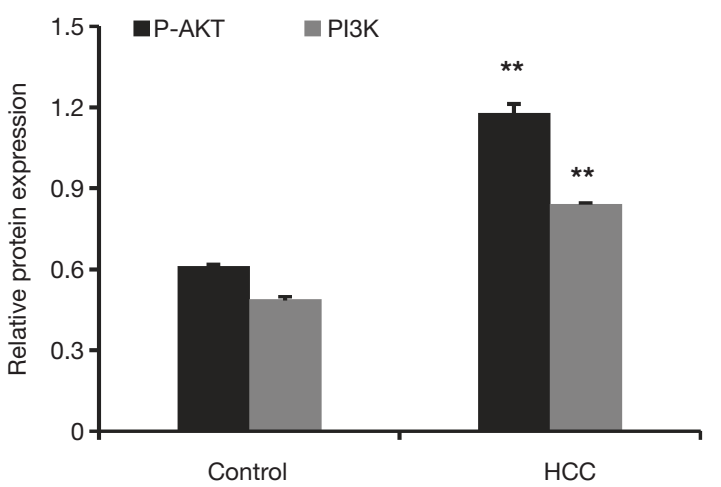

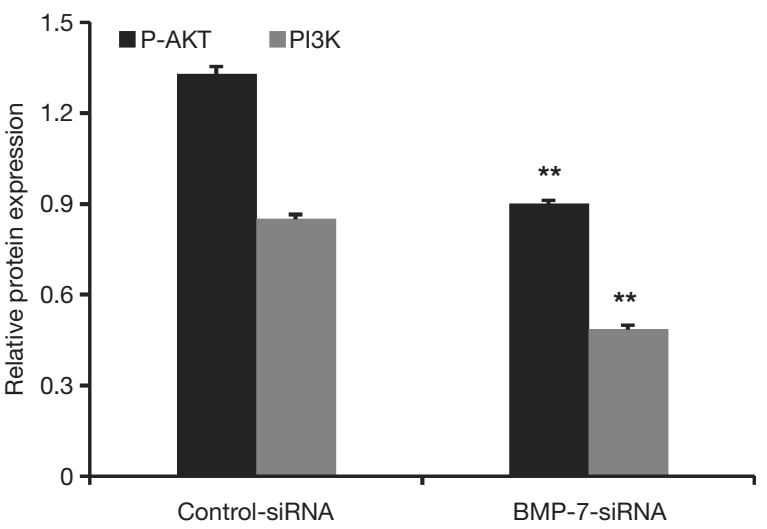

Figure 4 Effect of BMP-7 on the PI3K/AKT signaling pathway in human HCC tissue and HepG2 cells. (A) The protein expression of PI3K and P-AKT were detected by western blot in human HCC tissues and their controls. (B) The protein expression of PI3K and P-AKT were detected by western blot in HepG2 cells with BMP-7-siRNA and control-siRNA. Data are expressed as mean \pm SEM. **, P<0.01; and at least three independent experiments were performed in each panel. BMP-7, bone morphogenetic protein 7; HCC, hepatocellular carcinoma.

PI3K were detected by western blot. As shown in Figure 5 A, the level of P-AKT and PI3K protein was significantly decreased under administration of HP in a dose-dependent manner, and BMP-7 siRNA could abrogate the difference of AKT phosphorylation between cells with and without HP treatment (Figure 5B). In conclusion, all of the above results show that HP inhibits cell proliferation by reducing the BMP-7-dependent PI3K/AKT signaling pathway in HCC cells.

\section{Discussion}

In the current study, we found that HP inhibited the proliferation of HepG2 cells through cell cycle arrest and HP inhibited cell proliferation by down-regulating the BMP7/PI3K/AKT signaling. Current study suggests a novel treatment using $\mathrm{HP}$ as a potential therapy against HCC.
Previous studies have reported that BMPs family display significantly higher expression in tumors, which have been used as new biomarkers for the prognosis of cancer patients. In HCC, the member of BMPs (BMP-2, -4, -6, -7, -8, $-9,-10,-11,-13$, and -15$)$ revealed enhanced expression levels (21). In non-small cell lung cancer, BMP-2 level was increased in patients' serum, and was significantly correlated with poor prognosis. It can be regarded as an independent negative predictor for the prognosis of patients (6). Serum BMP-4 up-expression is closely associated with shorter patients' overall and disease-free survival, which serves as a pivotal marker for predicting the recurrence and prognosis of HCC patients after surgery (22). Recent studies have implied BMP-7 is present in cancers, including prostate, breast, and colon cancers, in which it is involved in regulating cancer cell proliferation $(9,10,23)$. Our current study further verified the increased expression of BMP-7 in 

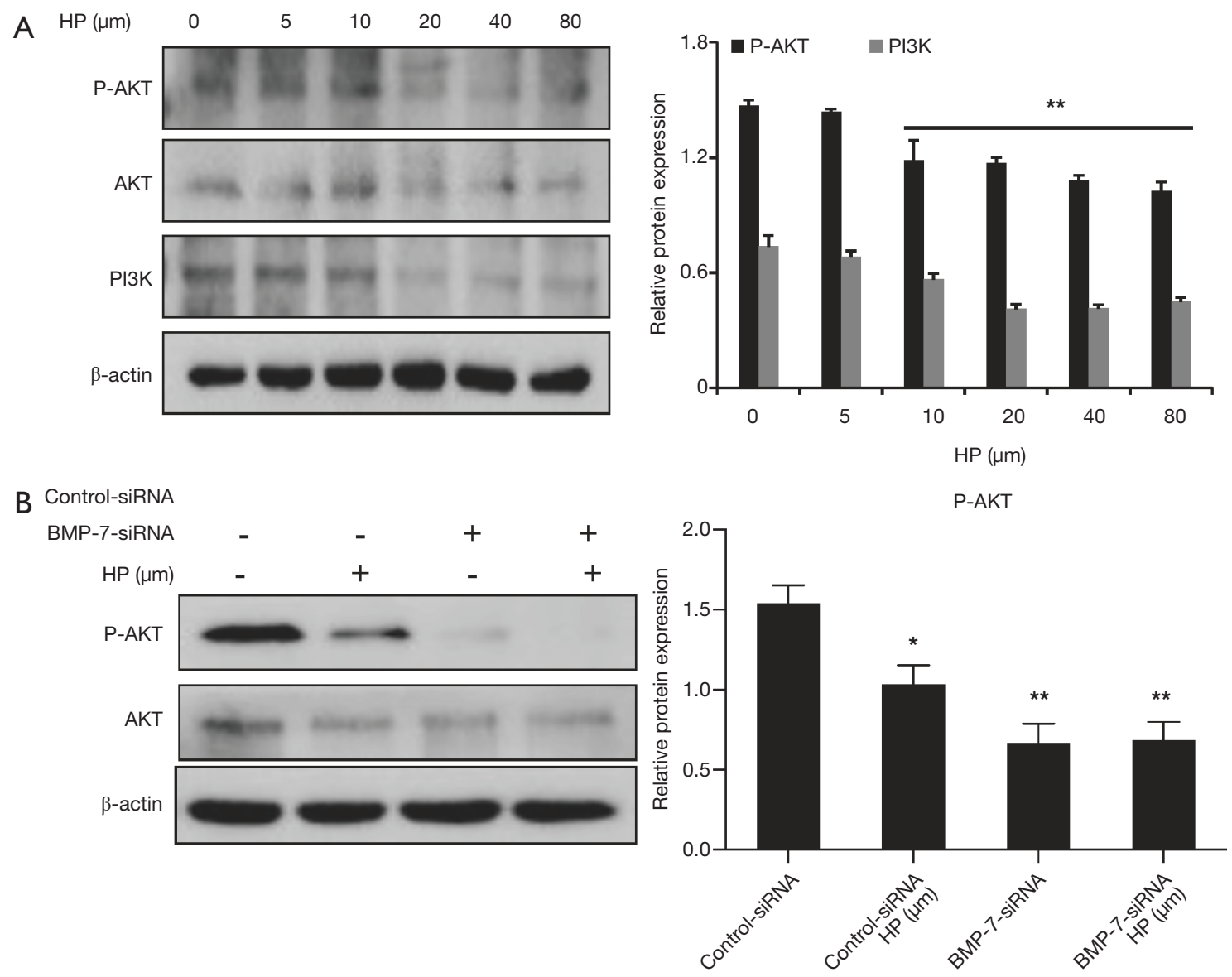

Figure 5 Effect of HP on the PI3K/AKT signaling pathway in HepG2 cells. (A) Cells were treated with HP for 24 hours, and the protein expression of PI3K and P-AKT were detected by western blot. (B) Cells were treated with or without HP (40 $\mu$ M) and with control-siRNA or BMP-7-siRNA for 24 hours, and the protein expression of P-AKT were detected by western blot. Data are expressed as mean \pm SEM. *, $\mathrm{P}<0.05$; **, $\mathrm{P}<0.01$; and at least three independent experiments were performed in each panel. HP, hyperoside; BMP-7, bone morphogenetic protein 7 .

human HCC tissues, and the silencing of BMP-7 restrained HepG2 cell proliferation in vitro. These data show that BMP-7 might be a promising target against human HCC.

BMP-7 is a secreted multifunctional protein, which has various biological activities. It participates in regulating cell proliferation, differentiation, and apoptosis, and plays a critical role in the formation of tissues and organs, the development of embryos, and the repair of injured tissues (24-26). Moreover, BMP-7, which belongs to the superfamily of TGF- $\beta$, has been implied to play a role in fibrogenesis (27). Chronic fibrotic livers eventually develop liver cirrhosis, which causes HCC, and further investigations are warranted to determine whether targeting BMP-7 in fibrotic or cirrhotic livers can prevent HCC.
HP is a bioactive component isolated from Ericaceae, Celastraceae and Guttifera. Previous studies have revealed that HP has a variety of pharmacological and biological effects including anti-oxidant, anti-viral, and anti-coagulant, especially anti-inflammatory activities (28-30). It has been reported to have remarkable anti-cancer effects $(19,31)$. Although HP has been implied to play a role in HCC cancer cells $(32,33)$, its direct effect has never been determined. We found HP inhibited HCC cancer cell proliferation in a dose-dependent manner, and administration of HP also led to a decline in BMP-7 expression in a dose-dependent manner. Since BMP-7 knockdown also plays an inhibitory role in HepG2 cell proliferation, this suggests that HP suppresses cell proliferation by targeting BMP-7 in HepG2 
cells.

The significance of the PI3K/AKT pathway and its potential as a therapeutic target in human cancers has been reported in several preclinical studies, including lung cancer (34), renal cancer (35), glioblastoma (36), breast cancer (37), and neuroblastoma (38). It has been reported that the PI3K/AKT signaling pathway plays a vital role in HCC and is activated in $30-55 \%$ of HCC cases (39). In our study, the expression of PI3K and P-AKT was markedly up-regulated in human HCC, which indicated that the mechanisms of BMP-7 involved activation of the PI3K/AKT signaling pathway in human HCC. We also discovered that HP exerted its effect through repressing the PI3K/AKT signaling pathway in HepG2 cells, and BMP-7 knockdown could not further inhibit AKT phosphorylation in HP-treated cells. This indicated that HP suppresses the PI3K/AKT pathway through down-regulating BMP-7.

Previous studies have indicated HP inhibits prostate cancer cell growth by regulation of microRNA-21 and microRNA-27a $(17,18)$. Yet the results of the present study benefit us to offer a novel insight into the mechanism of the inhibitory effect of HP on cell proliferation in human HCC and other cancers. Moreover, Huang et al. have previously reported that HP attenuates the inflammatory response by activating PPAR- $\gamma$ in lipopolysaccharide (LPS)treated macrophages (40). This indicated that HP plays an important role not only in cancer cells, but also in the microenvironment. Thus, further studies should pay close attention to the role of HP in cancer immunity in HCC.

In conclusion, HP, a flavonoid compound derived from a traditional Chinese medicine, suppressed the proliferation of HCC cancer cells by targeting BMP-7, accompanied by cell cycle arrest, and this anti-proliferating effect was mediated by the PI3K/AKT signaling pathway. Our study also highlights the role of BMP-7 in the proliferation of HCC cancer cells and suggests that BMP-7 is a new target against HCC. Our findings support the possibility of the application of flavonoid compounds in anti-HCC therapy, which might lead to new therapeutics for the treatment of HCC. In addition, it would be promising to develop the derivatives of HP according to its chemical structure and achieve the development of lead compounds with better anti-cancer activity.

\section{Acknowledgments}

Funding: This work was supported by grants from the Research and Development of Rehabilitation Related
Technology Zhejiang University-Fuchun Rehabilitation Joint Research Center (2020-KYY-518051-0066).

\section{Footnote}

Reporting Checklist: The authors have completed the MDAR reporting checklist. Available at https://dx.doi. org/10.21037/atm-21-2980

Data Sharing Statement: Available at https://dx.doi. org/10.21037/atm-21-2980

Conflicts of Interest: All authors have completed the ICMJE uniform disclosure form (available at https://dx.doi. org/10.21037/atm-21-2980). The authors have no conflicts of interest to declare.

Ethical Statement: The authors are accountable for all aspects of the work in ensuring that questions related to the accuracy or integrity of any part of the work are appropriately investigated and resolved. All procedures performed in this study were in accordance with the Declaration of Helsinki (as revised in 2013). Clinical Research Ethics Committee of the First Affiliated Hospital, College of Medicine, Zhejiang University approved the design of the study (IIT20210), and the patient sample study protocol. All patients provided signed informed consent.

Open Access Statement: This is an Open Access article distributed in accordance with the Creative Commons Attribution-NonCommercial-NoDerivs 4.0 International License (CC BY-NC-ND 4.0), which permits the noncommercial replication and distribution of the article with the strict proviso that no changes or edits are made and the original work is properly cited (including links to both the formal publication through the relevant DOI and the license). See: https://creativecommons.org/licenses/by-nc-nd/4.0/.

\section{References}

1. Kim E, Viatour P. Hepatocellular carcinoma: old friends and new tricks. Exp Mol Med 2020;52:1898-907.

2. Shin JW, Chung YH. Molecular targeted therapy for hepatocellular carcinoma: current and future. World J Gastroenterol 2013;19:6144-55.

3. Li N, Jiang S, Shi J, et al. Construction of a potential microRNA, transcription factor and mRNA regulatory network in hepatocellular carcinoma. Transl Cancer Res 
2020;9:5528-43.

4. Patel SR, Dressler GR. BMP7 signaling in renal development and disease. Trends Mol Med 2005;11:512-8.

5. Milán M. Sculpting a fly leg: BMP boundaries and cell death. Nat Cell Biol 2007;9:17-8.

6. Fei ZH, Yao CY, Yang XL, et al. Serum BMP-2 upregulation as an indicator of poor survival in advanced non-small cell lung cancer patients. Asian Pac J Cancer Prev 2013;14:5293-9.

7. Albilia JB, Tenenbaum HC, Clokie CM, et al. Serum levels of BMP-2, 4, 7 and AHSG in patients with degenerative joint disease requiring total arthroplasty of the hip and temporomandibular joints. J Orthop Res 2013;31:44-52.

8. Raida M, Clement JH, Ameri K, et al. Expression of bone morphogenetic protein 2 in breast cancer cells inhibits hypoxic cell death. Int J Oncol 2005;26:1465-70.

9. Alarmo EL, Rauta J, Kauraniemi P, et al. Bone morphogenetic protein 7 is widely overexpressed in primary breast cancer. Genes Chromosomes Cancer 2006;45:411-9.

10. Miyazaki H, Watabe T, Kitamura T, et al. BMP signals inhibit proliferation and in vivo tumor growth of androgen-insensitive prostate carcinoma cells. Oncogene 2004;23:9326-35.

11. Li X, Sun B, Zhao X, et al. Function of BMP4 in the formation of vasculogenic mimicry in hepatocellular carcinoma. J Cancer 2020;11:2560-71.

12. Yuan YM, Ma N, Zhang EB, et al. BMP10 suppresses hepatocellular carcinoma progression via PTPRS-STAT3 axis. Oncogene 2019;38:7281-93.

13. Buijs JT, Henriquez NV, van Overveld PG, et al. TGFbeta and BMP7 interactions in tumour progression and bone metastasis. Clin Exp Metastasis 2007;24:609-17.

14. Boon MR, van der Horst $G$, van der Pluijm G, et al. Bone morphogenetic protein 7: a broad-spectrum growth factor with multiple target therapeutic potency. Cytokine Growth Factor Rev 2011;22:221-9.

15. Alarmo EL, Pärssinen J, Ketolainen JM, et al. BMP7 influences proliferation, migration, and invasion of breast cancer cells. Cancer Lett 2009;275:35-43.

16. Li W, Cai HX, Ge XM, et al. Prognostic significance of BMP7 as an oncogene in hepatocellular carcinoma. Tumour Biol 2013;34:669-74.

17. Yang FQ, Liu M, Li W, et al. Combination of quercetin and hyperoside inhibits prostate cancer cell growth and metastasis via regulation of microRNA-21. Mol Med Rep 2015;11:1085-92.

18. Li W, Liu M, Xu YF, et al. Combination of quercetin and hyperoside has anticancer effects on renal cancer cells through inhibition of oncogenic microRNA-27a. Oncol Rep 2014;31:117-24.

19. Li FR, Yu FX, Yao ST, et al. Hyperin extracted from Manchurian rhododendron leaf induces apoptosis in human endometrial cancer cells through a mitochondrial pathway. Asian Pac J Cancer Prev 2012;13:3653-6.

20. Li B, Li J, Xu WW, et al. Suppression of esophageal tumor growth and chemoresistance by directly targeting the PI3K/AKT pathway. Oncotarget 2014;5:11576-87.

21. Maegdefrau U, Bosserhoff AK. BMP activated Smad signaling strongly promotes migration and invasion of hepatocellular carcinoma cells. Exp Mol Pathol 2012;92:74-81.

22. Guo X, Xiong L, Zou L, et al. Upregulation of bone morphogenetic protein 4 is associated with poor prognosis in patients with hepatocellular carcinoma. Pathol Oncol Res 2012;18:635-40.

23. Beck SE, Jung BH, Del Rosario E, et al. BMP-induced growth suppression in colon cancer cells is mediated by p21WAF1 stabilization and modulated by RAS/ERK. Cell Signal 2007;19:1465-72.

24. Weiskirchen R, Meurer SK. Bone morphogenetic protein-7 in focus: a member of the transforming growth factor-beta superfamily is implicated in the maintenance of liver health. Hepatology 2007;45:1324-5.

25. Hao ZM, Cai M, Lv YF, et al. Oral administration of recombinant adeno-associated virus-mediated bone morphogenetic protein-7 suppresses $\mathrm{CCl}(4)$-induced hepatic fibrosis in mice. Mol Ther 2012;20:2043-51.

26. Luo DD, Phillips A, Fraser D. Bone morphogenetic protein-7 inhibits proximal tubular epithelial cell Smad3 signaling via increased SnoN expression. Am J Pathol 2010;176:1139-47.

27. Zhao XK, Cheng ML, Wu RM, et al. Effect of Danshao Huaxian capsule on Gremlin and bone morphogenetic protein-7 expression in hepatic fibrosis in rats. World J Gastroenterol 2014;20:14875-83.

28. Bernatoniene J, Trumbeckaite S, Majiene D, et al. The effect of crataegus fruit extract and some of its flavonoids on mitochondrial oxidative phosphorylation in the heart. Phytother Res 2009;23:1701-7.

29. Havsteen BH. The biochemistry and medical significance of the flavonoids. Pharmacol Ther 2002;96:67-202.

30. Middleton E Jr, Kandaswami C, Theoharides TC. The effects of plant flavonoids on mammalian cells: implications for inflammation, heart disease, and cancer. Pharmacol Rev 2000;52:673-751. 
31. Zhang N, Ying MD, Wu YP, et al. Hyperoside, a flavonoid compound, inhibits proliferation and stimulates osteogenic differentiation of human osteosarcoma cells. PLoS One 2014;9:e98973.

32. Han J, Meng J, Chen S, et al. YY1 complex promotes quaking expression via super-enhancer binding during EMT of hepatocellular carcinoma. Cancer Res 2019;79:1451-64.

33. Sun Y, Pan Z, Yang C, et al. Comparative assessment of phenolic profiles, cellular antioxidant and antiproliferative activities in ten varieties of sweet potato (Ipomoea Batatas) storage roots. Molecules 2019;24:4476.

34. Cui D, Feng Y, Shi K, et al. Long non-coding RNA TRPM2-AS sponges microRNA-138-5p to activate epidermal growth factor receptor and PI3K/AKT signaling in non-small cell lung cancer. Ann Transl Med 2020;8:1313.

35. Sourbier C, Lindner V, Lang H, et al. The phosphoinositide 3-kinase/Akt pathway: a new target in human renal cell carcinoma therapy. Cancer Res 2006;66:5130-42.

Cite this article as: Wei S, Sun Y, Wang L, Zhang T, Hu W, Bao W, Mao L, Chen J, Li H, Wen Y, Chen Z. Hyperoside suppresses BMP-7-dependent PI3K/AKT pathway in human hepatocellular carcinoma cells. Ann Transl Med 2021;9(15):1233. doi: 10.21037/atm-21-2980
36. Weber GL, Parat MO, Binder ZA, et al. Abrogation of PIK3CA or PIK3R1 reduces proliferation, migration, and invasion in glioblastoma multiforme cells. Oncotarget 2011;2:833-49.

37. Zhou J, Xiang AZ, Guo JF, et al. miR-30b suppresses the progression of breast cancer through inhibition of the PI3K/Akt signaling pathway by targeting Derlin-1. Transl Cancer Res 2019;8:180-90.

38. Opel D, Naumann I, Schneider M, et al. Targeting aberrant PI3K/Akt activation by PI103 restores sensitivity to TRAIL-induced apoptosis in neuroblastoma. Clin Cancer Res 2011;17:3233-47.

39. Villanueva A, Chiang DY, Newell P, et al. Pivotal role of mTOR signaling in hepatocellular carcinoma. Gastroenterology 2008;135:1972-83, 1983.e1-11.

40. Huang C, Yang Y, Li WX, et al. Hyperin attenuates inflammation by activating PPAR- $\gamma$ in mice with acute liver injury (ALI) and LPS-induced RAW264.7 cells. Int Immunopharmacol 2015;29:440-7.

(English Language Editor: B. Draper) 